\title{
Virtual Reality Image Processing for Effective Deep Brain Stimulation of the Anterior Thalamic Nucleus in Epilepsy Patients
}

\author{
Crystal N Adams, MD', Mohamad Z Koubeissi, MD², Aalap Herur-Raman ${ }^{1}$ and Donald C Shields, MD, \\ PhD $^{1^{*}}$
}

${ }^{1}$ Department of Neurosurgery, The George Washington University, Washington, DC USA

${ }^{2}$ Department of Neurology, The George Washington University, Washington, DC USA

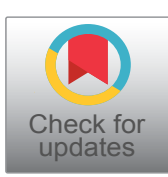

*Corresponding author: Donald C Shields, MD, PhD, Department of Neurosurgery, The George Washington University, 2150 Pennsylvania Ave., NW, Ste. 7-409, Washington, DC 20037, Tel: 202-741-2750, Fax: 202-741-2742

\begin{abstract}
Deep brain stimulation of the anterior thalamic nucleus for treatment of pharmacoresistant epilepsy is a surgical option for patients who are not candidates for resective surgery. One of the challenges of this technique relates to the small size of the anterior thalamic nucleus (approximately $10 \mathrm{~mm}$ $\times 5 \mathrm{~mm} \times 5 \mathrm{~mm}$ ). Stimulation several millimeters outside the intended target provides suboptimal seizure control. One option for achieving accurate stimulation includes the use of virtual reality software image processing techniques. This report describes the effective use of pre- and post-operative image processing to achieve optimal results in a patient treated with anterior thalamic nucleus deep brain stimulation.
\end{abstract}

\section{Keywords}

Deep brain stimulation, Epilepsy, Thalamus, Virtual reality

\section{Introduction}

Epilepsy is one of the most common neurological disorders, affecting 50-70 million people worldwide. Approximately a third of this population is poorly responsive to antiepileptic drugs [1]. Medically-intractable epilepsy is defined by the failure of two appropriately selected and adequately dosed antiepileptic medications to achieve seizure control [2]. Intractable epilepsy is known to be associated with diminished health-related quality of life, increased psychological and cognitive comorbidities, as well as significant financial and psycho- social consequences [3].

While surgical resection is the gold standard intervention in patients who have an identifiable seizure focus, many patients are not ideal surgical candidates. These include patients with multifocal seizures or those with a seizure focus overlapping eloquent brain areas [4]. In these patients, neuromodulation therapy such as vagal nerve stimulation, responsive closed loop stimulation, or Deep Brain Stimulation (DBS) may be considered as an alternative.

Multiple structures including the cerebellum and thalamus (both centromedian and anterior thalamic nuclei) have been studied as potential targets for DBS in patients with medication resistant epilepsy. Since the 1970s, when early DBS studies in epilepsy were done, most reports have been limited by small numbers and variable results [4]. Three types of target sites have been described including the suspected seizure focus (e.g., hippocampus), deep subcortical structures (nucleus accumbens, caudate, subthalamic nucleus), and deep fiber bundles connected to specific brain regions [4]. Anterior thalamic nuclei stimulation falls into the second category and is thought to modulate neuronal networks like the Circuit of Papez which has been implicated in both emotional response processing and seizure propagation [5]. As a result, it is postulated that anterior thalamic stimulation may inhibit spread of sei-

Citation: Adams CN, Koubeissi MZ, Herur-Raman A, Shields DC (2020) Virtual Reality Image Processing for Effective Deep Brain Stimulation of the Anterior Thalamic Nucleus in Epilepsy Patients. Neurosurg Cases Rev 3:047. doi.org/10.23937/2643-4474/1710047

Accepted: November 26, 2020; Published: November 28, 2020

Copyright: (C) 2020 Adams CN, et al. This is an open-access article distributed under the terms of the Creative Commons Attribution License, which permits unrestricted use, distribution, and reproduction in any medium, provided the original author and source are credited. 
zure activity to associated cortical areas [4]. The SANTE trial, a double-blind randomized control trial assessing the efficacy of anterior thalamic stimulation in drug resistant epilepsy, demonstrated a significant long-term reduction of seizure frequency of stimulated subjects compared to the non-stimulated group $[6,7]$. The U.S. Food and Drug Administration recently approved this procedure for use in specific medication resistant seizure patients.

For epileptologists and surgeons, one remaining challenge with anterior thalamic DBS is the small size of the intended target nucleus; stimulation several millimeters outside the target is less effective for seizure prevention. Historically, DBS surgery techniques have utilized stereotactic planning wherein the target structure, which may not be easily seen on a patient's imaging, is defined using coordinates relative to readily identifiable anatomical structures (i.e., the anterior and posterior commissures). However, potential anatomic variability and surgery-related pneumocephalus are significant limitations of this method. Improved imaging quality from stronger magnetic fields such as 3T MR imaging better allows the clinician to directly visualize a structure of interest to improve target accuracy [8]. With the recent introduction of virtual reality techniques in neurosurgery, there is the potential to further augment the accuracy of target stimulation. As demonstrated in this report describing a patient undergoing anterior thalamic DBS for drug resistant epilepsy, virtual reality software can be employed to pre- and postoperative imaging studies to reduce electrode artifact and confirm accurate contact placement for effective stimulation.

\section{Case Report}

This patient is a 35-year-old right-handed man who was diagnosed with epilepsy at age 11 when he presented with a generalized tonic-clonic seizure; however, earlier staring spells were observed by family members. He received multiple antiepileptic medications including lacosamide and valproic acid without adequate seizure control. He underwent implantation of a vagal nerve stimulator without improvement in seizure frequency or severity. His brain MRI and FDG-PET were unremarkable, and his ictal SPECT suggested a seizure onset zone in the right temporal lobe with spread to the left temporal lobe. His surgical evaluation at another hospital included implantation of depth and strip electrodes, which revealed seizures with possible right frontal onset and rapid propagation to the left hippocampus. Other seizures appeared to originate from the left anterior frontal and left lateral frontal regions. Semiologically, three seizure types were described including hyper motor seizures involving both arms, staring spells, and vocalization followed by violent clonic movements. He then presented for surgical evaluation in 2018 at our institution and underwent depth electrode implantation in bilateral frontal and temporal regions, without identi- fication of the seizure focus. He was transitioned to triple therapy with lacosamide, clobazam, and perampanel with some noted improvement in seizure frequency. The patient and his family hoped for better seizure control, and anterior thalamic DBS was recommended.

On the morning of surgery, the patient was placed in a Radionics CRW stereotactic frame (Integra Life Sciences Services, Saint Priest, France) and taken for a stereotactic head CT scan with the frame in place. His preoperative MRI was loaded onto the Stealth Station system (Medtronic, Inc., Minneapolis, MN) and merged with the stereotactic CT for surgical planning. Precision VR software (Surgical Theater, Inc., Cleveland, $\mathrm{OH}$ ) was used to create a 360-degree Virtual Reality (VR) reconstruction of the patient's brain with the anterior thalamic nuclei, mammilothalamic tracts, and major blood vessels identified using the patient's preoperative imaging. This VR model was used in conjunction with the Stealth Neuronavigation software to plan the target and entry points (on the left and right frontal bones) as well as the electrode trajectories. These were designed to avoid the thalamostriate veins along the surface of the thalamus, any large arteries or veins along the electrode path, and the choroid plexus (since a transventricular trajectory was chosen). Entry points were near the coronal suture and approximately $2 \mathrm{~cm}$ lateral to the midline on each side. The stereotactic $x, y, z$, ring, and arc coordinates were then obtained for both approaches from the Stealth Neuronavigation software. After the patient was positioned, prepped, and draped in the standard fashion, the CRW frame was set to the coordinates for the left sided target. A small incision was made after shaving and infiltrating the scalp with local anesthetic. A burr hole was then created with a pneumatic drill assembly, and the dura was opened in a cruciate fashion. The left side was chosen for initial electrode placement since the thalamostriate vein on that side was within $2 \mathrm{~mm}$ of the proposed entry point into the thalamus. Pneumocephalus-related brain shift may have placed the vein in the path of the cannula if the right side was entered first. The cannula was passed down to $4 \mathrm{~mm}$ above target (just deep to the ventricular surface of the thalamus). Then the cannula stylet was removed and the tip of the 3389 DBS electrode (Medtronic, Inc., Minneapolis, MN) was inserted slightly beyond target - the rostral extent of the left mammilothalamic tract as it abuts the ventral posterior border of the anterior thalamic nucleus. The portion of the 3389 electrode with contacts near the tip is longer than the anterior thalamic nucleus, so only some of the contacts can be used for stimulation of the nucleus. Thus, contacts 0 and 1 were inserted deep to the target, while 2 and 3 were available for stimulation of the anterior thalamic nucleus (Figure 1). The electrode was secured in place at the burr hole site. A lateral skull X-ray was obtained to confirm position. The same procedure was performed on the right side. The neurology team then turned on the stimula- 

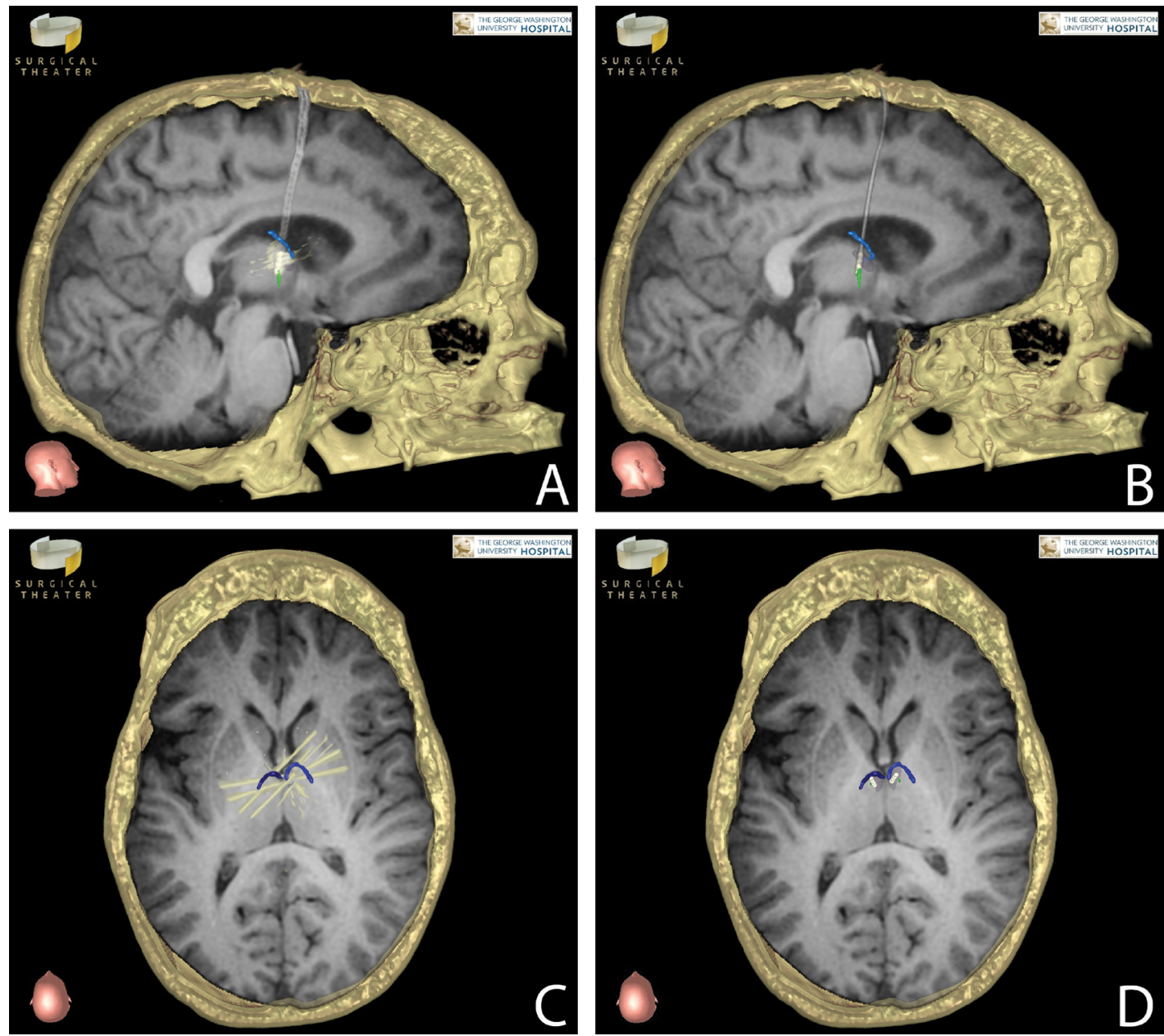

Figure 1: Each panel represents preoperative stereotactic MRI T1 sequences merged with postoperative CT scans (axial images are not inverted horizontally). Right DBS electrode in sagittal view (A) Prior to 3D grey-level mapping compared with post-mapping image; (B) Mammillothalamic tract (green); (C) Corresponding axial views before and after; (D) Grey-level mapping demonstrate the DBS electrodes in relation to the anterior thalamic nucleus (shaded dark gray). Metallic artifact (white) from the CT scan related to the 4 contacts is shown in panels A and C; this artifact obscures the anterior thalamic nucleus in panel A. The electrode diameter is likewise larger with CT-related distortion in panel A. Portions of the thalamostriate veins (blue) near the electrodes in axial views demonstrate the necessity of slightly more posterior placement of the left electrode, compared to the right, in order to avoid traversing the left thalamostriate vein.

tion at all 4 contacts bilaterally above 5 volts to confirm the patient did not have any new mood symptoms or neurological changes. The incisions were then closed in a standard fashion. The infraclavicular pulse generator was inserted during a different admission, and a postoperative head CT with stereotactic navigation protocol was obtained to confirm electrode placement. This CT was merged with the preoperative MRI using Precision VR software (Surgical Theater, Inc., Cleveland, OH). CT number 3D grey-level mapping was performed on this software in order to mitigate the typical contact-associated artifact seen on postoperative DBS imaging. Thus, the Neurology team could visually confirm contacts 2 and 3 were appropriate for stimulation.

\section{Discussion}

Recent technological advances have changed the way we learn and practice medicine. Virtual Reality (VR)-based simulation is on the forefront of these new technologies. VR software takes information from volumetric $2 \mathrm{D}$ patient images and creates an interactive $3 \mathrm{D}$ reconstruction. This 3D model allows for better understanding of the complex spatial relationships between various structures [9]. These tools can then be used to determine and plan the best surgical approach, while clearly identifying key nearby structures. These virtual reality platforms can be used in conjunction with standard stereotactic navigation software to augment the surgical planning experience [9]. In addition, in many surgical subspecialties, virtual reality has been introduced as a method for both teaching and assessing surgical skills. While the use of virtual reality software has been previously described in other surgical and medical subspecialties, its use is relatively new in neurosurgery [10].

The adaptation of virtual reality software in preoperative surgical planning has been previously described, particularly in cases of tumor resection. However, in the case of this patient, virtual reality software was used in the preoperative, intraoperative and postoperative settings. Prior to surgery, virtual reality software was used to create a 3D reconstruction identifying key 
structures and fiber tracts necessary for more accurate target identification and trajectory planning. This model was used in conjunction with stereotactic navigation software to plan trajectories for electrode insertion. As previously noted, DBS has typically included indirect targeting, wherein, the target of interest is defined by coordinates relative to the anterior and posterior commissures. However, virtual reality software helps overcome anatomic variability limits to the accuracy of this method with improved target border visualization, especially when the target structure is smaller than the distance spanning the proximal-to-distal stimulating electrode contacts.

In the post-operative setting, virtual reality software was used to fuse post-operative stereotactic imaging with the interactive $3 \mathrm{D}$ reconstruction to identify the locations of each of the four DBS electrode contacts (free of metallic artifact) within and adjacent to the target. This information was then used to help guide postoperative therapy by providing the patient's neurologist with guidance regarding which contacts may be most useful for stimulation. This is particularly helpful when pneumocephalus following creation of the burr hole causes brain shift and movement of the small anterior thalamic nucleus target. Future enhancements of 3D visualization of the target and stimulating contacts may thus allow for greater stimulation efficacy and seizure prevention.

\section{Conclusions}

Anterior thalamic DBS has been described as a therapeutic option for patients with medication resistant epilepsy which is not amenable to curative surgical resection. Since the anterior thalamic nucleus is a small target for DBS, virtual reality software may be used to improve the accuracy of target identification and trajectory planning in the preoperative setting. In addition, it can be employed in the postoperative setting to identify electrode contacts which are most effective for therapeutic stimulation.

\section{References}

1. Singh A, Trevick S (2016) The epidemiology of global epilepsy. Neurol Clin 34: 837-847.

2. Kwan P, Arzimanoglou A, Berg AT, Brodie MJ, Allen Hauser W, et al. (2010) Definition of drug resistant epilepsy: Consensus proposal by the ad hoc task force of the ILAE commission on therapeutic strategies. Epilepsia 51: 10691077.

3. Johnson EK, Jones JE, Seidenberg M, Hermann BP (2004) The relative impact of anxiety, depression, and clinical seizure features on health-related quality of life in epilepsy. Epilepsia 45: 544-550.

4. Li MCH, Cook MJ (2018) Deep brain stimulation for drug-resistant epilepsy. Epilepsia 59: 273-290.

5. Montgomery EB, Gale JT (2008) Mechanisms of action of deep brain stimulation (DBS). Neurosci Biobehav Rev 32: 388-407.

6. Piacentino M, Durisotti C, Garofalo PG, Bonanni P, Volzone A, et al. (2015) Anterior thalamic nucleus deep brain Stimulation (DBS) for drug-resistant complex partial seizures (CPS) with or without generalization: Long-term evaluation and predictive outcome. Acta Neurochirurgica 157: 1525-1532.

7. Vicenta S, Witt T, Worth R, Henry TR, Gross RE, et al. (2015) Long-term efficacy and safety of thalamic stimulation for drug-resistant partial epilepsy. Neurology 84: 10171025.

8. Buentjen L, Kopitzki K, Schmitt FC, Voges J, Tempelmann C, et al. (2014) Direct targeting of the thalamic anteroventral nucleus for deep brain stimulation by T1-weighted magnetic resonance imaging at $3 \mathrm{~T}$. Stereotactic and Functional Neurosurgery 92: 25-30.

9. Bernardo A (2017) Virtual reality and simulation in neurosurgical training. World Neurosurg 106: 1015-1029.

10. Pelargos PE, Nagasawa DT, Lagman C, Tenn S, Demos JV, et al. (2017) Utilizing virtual and augmented reality for educational and clinical enhancements in neurosurgery. $J$ Clin Neurosci 35: 1-4. 